\title{
A MT-CDMA Based Wireless Body Area Network for Ubiquitous Healthcare Monitoring
}

\author{
Jui-Yuan Yu, Wan-Chun Liao, and Chen-Yi Lee \\ Department of Electronics Engineering, National Chiao-Tung University, Taiwan R.O.C. \\ Email: \{blues,cylee\}@royals.ee.nctu.edu.tw
}

\begin{abstract}
This paper presents a multi-tone CDMA (MT-CDMA) based system specification for wireless body area network (WBAN) applications. According to the factors of bandwidth, carrier frequency, and electric field intensity defined in wireless medical telemetry service, the function blocks and data format are designed through the considerations of hardware cost, power consumption, and system performance. The design constraints for baseband processor, data conversion, and RF circuits are defined. This work achieves $P E R=1 \%$ and allows interferer distance less than $1.01 \mathrm{~m}$. In energy-spectrum efficiency, it provides 3.125 times energy-spectrum product less than the state-of-the-art systems for healthcare applications.
\end{abstract}

Index Terms-CDMA, OFDM, WBAN

\section{INTRODUCTION}

U BIQUITOUS healthcare monitoring plays a crucial role in physical status tracking and recording. This extends medical services from the closed in-hospital systems to any open roaming spaces.

The wireless body area network (WBAN) is specifically designed for the applications of body signal gathering and monitoring to provide reliable physical information. Combined with variant sensor functions, WBAN manages signal communications from different body area through a wireless channel for body condition monitoring or diagnosis. Those include surface measurements, like ECG, EEG, and body temperature, and chip-implanted body status inspections, like blood glucose-level observation and stomach capsule. WBAN requires long-term stability in terms of operation duration and signal integrity, especially for those chip-implanted applications. Existing candidate systems which may provide the medical or healthcare services are found in IEEE 802.15.4 LR-WPAN (or ZigBee) [1], impulse-radio ultra-wideband (IR-UWB) [2], and IEEE 802.15.1 WPAN (or Bluetooth) [3]. These solutions are designed for widespread applications, including entertainment, positioning, factory product management, and healthcare. For applications which require image or large file transfer, IEEE 802.11a WLAN [4] offers a

Work supported by MOEA and NSC of Taiwan, ROC, under Grant 94-EC-17-A-03-S1-0005 and NSC-94-2220-E-009-033- respectively linking range and data rate of $100 \mathrm{~m}$ and $54 \mathrm{Mb} / \mathrm{s}$, respectively, which performs a long range connection and large data exchange.

With the increasing demands in wireless body-area applications, however, those candidate systems have difficulties in performing consumer electronic device functions and healthcare applications at the same time. As a result, more and more attentions are paid in the WBAN system construction for body-oriented applications. To meet the long-term stability, a system is constrained by $\mu$-level power dissipation, multiple-sensor/user coexistence capability, and interference rejection ability. To reach extreme low power performance, an asymmetric communication network is required. This means the hardware cost and power consumption in a transmitter are designed as little as possible, and a robust receiver is required to achieve high performance wireless communications. The transmission range is usually designed no more than 3 meters, but the data transmission reliability is highly concerned. According to the Federal Communications Commission (FCC) spectrum specification [5], it allocates three sets of frequency band $(611 \mathrm{MHz}, 1.4 \mathrm{GHz}$, and $1.43 \mathrm{GHz})$ in the U.S. for the wireless medical telemetry service (WMTS). It provides an interference-free spectrum to prevent from the operation in a crowded industrial-science-medical (ISM) band. Following the WMTS constraints, we propose a multi-tone CDMA (MT-CDMA) [13] based wireless system scheme to enable the applications of ubiquitous healthcare monitoring.

The paper is organized as follows. Section II defines the system specification according to WMTS and the coexistence operation scenario. Then a corresponding performance evaluation and comparison are explored in section III. Finally, we conclude our work in section IV.

\section{SYSTEM SPECIFICATION}

The WBAN operations depending on the sensor number in a function unit could be divided into two categories: single-sensor link (SSL) and multiple-sensor link (MSL) as shown in Fig. 1. For those functions of SSL networks, it requires a single wireless sensor node (WSN) to gather body physical information in a wireless manner to a remote central process node (CPN). Such application could be found for 
example in temperature sensing or blood glucose-level inspection. On the other hand, a MSL network acquires body information by a multiple of WSN, separately distributed in the concerned body area. For example, a wireless ECG system requires four to twelve WSNs depending on the necessary measurement accuracy, which allocate in the surrounding of human's heart and feet.

For a variety of sensor categories, a user may enable more than one function unit at the same time to perform the health monitoring in different body areas, which could be any combinations of SSL and MSL networks. To enable the network operating in public that each user constitutes his/her own transmission links with coexistence capability, we propose a MT-CDMA based WBAN with time division multiplexing (TDM) to achieve this goal. The assignment of user (spreading) code is on a function-unit basis rather than a sensor node basis. When a certain function unit belongs to the MSL, this set of WSNs are assigned only one user code but transmitted in an exclusive time slot by the TDM control to maximize the capacity of co-operation sensors. Fig. 2 illustrates a transmission example of joint SSL and MSL in a multiple user scenario. Each function unit has its own operation duration depending on the body signal characteristics. All of them are transmitted in a burst, and a remote device is able to identify each function unit in an interference environment.

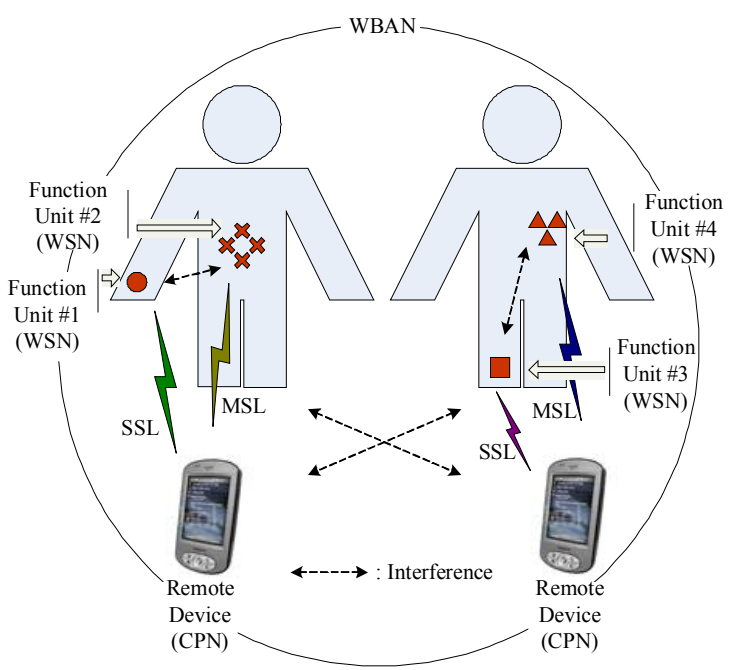

Fig. 1. A WBAN allows a variant number of users, central process nodes (CPN), wireless sensor nodes (WSN), and function units. The communication behavior belongs to either single-sensor node (SSL) or multiple-sensor node (MSL).

A spreading code length determines a maximum number of function units in the network and also limits the transmission throughput in an allowable spectrum bandwidth. Therefore, the WMTS criterion is examined to have the tradeoff between operation user number and transmission data throughput. WMTS allocates three spectrum band, $611 \mathrm{MHz}, 1.4 \mathrm{GHz}$, and $1.43 \mathrm{GHz}$, which allows a maximum signal bandwidth of $6 \mathrm{MHz}$, $5 \mathrm{MHz}$, and $3 \mathrm{MHz}$, respectively. Assume the $1.4 \mathrm{GHz}$ spectrum band is used in our proposed work, then the maximum achievable bandwidth after signal spreading is $5 \mathrm{MHz}$. To enable a 10-function-unit cooperation in the network, the Gold code [6] with spreading code length 31 is applied. As a result, a maximum achievable symbol rate before user-code spreading is $5 \mathrm{M} / 31=161.29 \mathrm{kS} / \mathrm{s}$ (if the spectrum mask is not considered). So, the upper bound of the symbol rate and the number of function units are now determined by the available spectrum bandwidth and the user-code length, respectively.

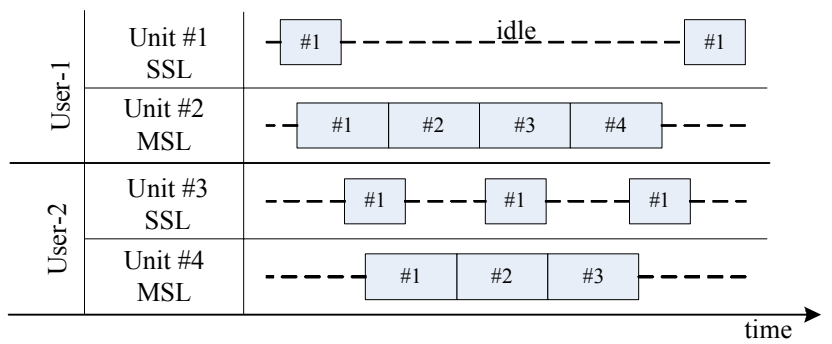

Fig. 2. An example of data transmission with both SSL and MSL sensor nodes with variant operation duty cycle.

In Fig. 3, we depict the proposed function blocks in a transmitter. The body signals come from a WSN. Depending on variant application requirements, an optional data compression or encryption could be added in signal processing. For the WBAN applications, it is required to have extreme low power consumption in a transmitter because a transmitter is made as small as possible for wearability, and is usually energy and battery limited. To achieve this goal, a function block of conjugate-symmetric subcarrier spreading is added to reduce the two-path transmission circuits (in-phase and quadrature-phase paths) to a single-path one. For example, assume the input subcarriers to an inverse fast Fourier transformation (IFFT) has the form of

$$
S=\left\{0, d_{N / 2+1}, d_{N / 2+2}, \ldots, d_{1}, 0, d_{1}^{*}, \ldots, d_{N / 2-2}^{*}, d_{N / 2-1}^{*}\right\}
$$

where $d$ is the frequency-domain subcarrier and $N$ is the IFFT block size. So, it is derived that

$$
s=\operatorname{IFFT}\{S\}_{N}=\operatorname{Re}\{s\}
$$

In other words, every input pattern with the form of (1) results in a zero imaginary part of the IFFT output. This equivalently saves one path of DAC and RF circuits, and thus the corresponding power consumption and hardware cost are reduced. Those subcarriers in set $S$ are duplicated one time, and this is also considered as spectrum spreading with spreading factor two. In a constellation mapper, it modulates signals into QPSK, and a higher order modulation more than 16-QAM is undesired in the system to prevent from the 


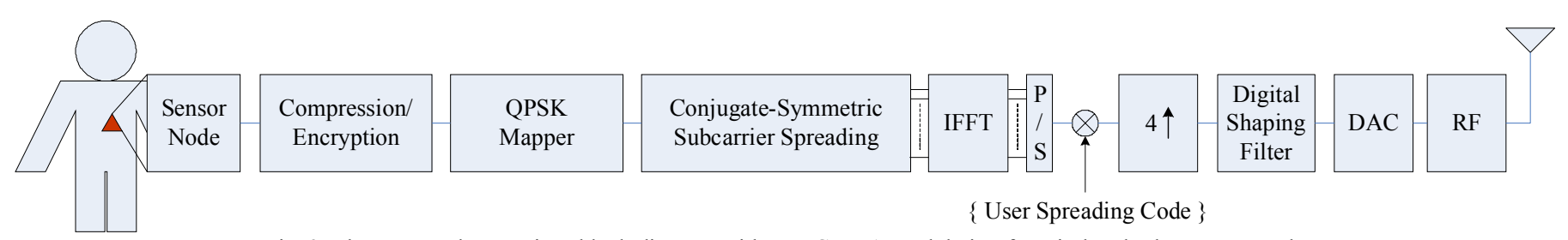

Fig. 3. The proposed transmitter block diagram with MT-CDMA modulation for wireless body area network.

peak-to-average-power ratio (PAPR) problems. Considering the robustness to a fading channel and PAPR problems, a 16-point IFFT is used for OFDM modulation. The guard interval with $1 / 8$ OFDM block size is applied prior to each OFDM symbol. Therefore, the system achieves the equalization ability to a fading channel. This low-order constellation mapper and small block size IFFT reduce the PAPR problem with the aid of digital reduction techniques [7][8] to achieve mild signal distortion and low out-of-band spurious interference. Then the IFFT output is spread by the spreading code and sent to a DAC. Finally, the word-length of DAC circuit decides the computation complexity and system performance, and it will be addressed in the simulation results later.

In the signal power spectrum, WMTS has strict radiation power intensity definition. In the low band, $608-614 \mathrm{MHz}$, the maximum in-band and out-of-band electrical field strength is defined as $200 \mathrm{mV} / \mathrm{m}$ and $200 \mathrm{uV} / \mathrm{m}$, respectively. In the high band, $1395-1400 \mathrm{MHz}$ and $1429-1432 \mathrm{MHz}$, the maximum in-band and out-of-band field strength is $740 \mathrm{mV} / \mathrm{m}$ and $540 \mathrm{uV} / \mathrm{m}$, respectively. Both the high and low band has the field strength measured at a 3-meter distance. As a result, a signal spectrum mask is specified in Fig. 4. This equivalently defines the allowable bandwidth for baseband signal transmission, i.e. $3 \mathrm{MHz}$. To meet this spectrum mask design challenge, the digital signals before DAC circuit are upsampled four times and applied to a digital shaping filter to balance the power and design challenges between the RF front-end and baseband circuits.

\section{SYSTEM EVALUATIONS AND COMPARISON}

The evaluation platform has the specification of OFDM modulation with CDMA identification. In order to provide sufficient information to reflect the system performance and design the wireless communications link budget, we assume ten function units are in cooperation. To define the system's worst operation condition, however, we assume a fully coexistence of those ten devices without any idle time. In other words, the other nine WSNs appear to be the background noise or interferers to a certain sensor unit. In a $N$-WSN $(N=2 \sim 10)$ coexistence environment, the rest $(N-1)$-WSNs act as the interferers to a certain WSN-CPN transmission link. Therefore, Fig. 5 shows simulation performance in terms of the minimum distance of $(N-1)$-WSN interferers to a certain CPN, in which the desired WSN-CPN maintains transmission link with packet error rate $(\mathrm{PER})=1 \%$. Because our proposed system is

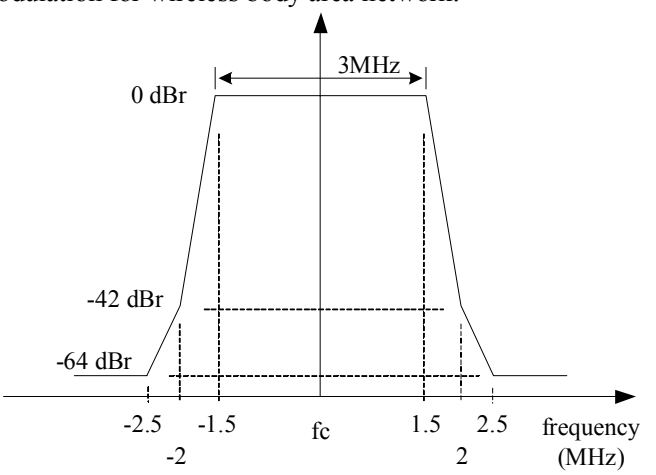

Fig. 4. The designed passband power spectrum mask and the required radiation power level defined in WMTS.

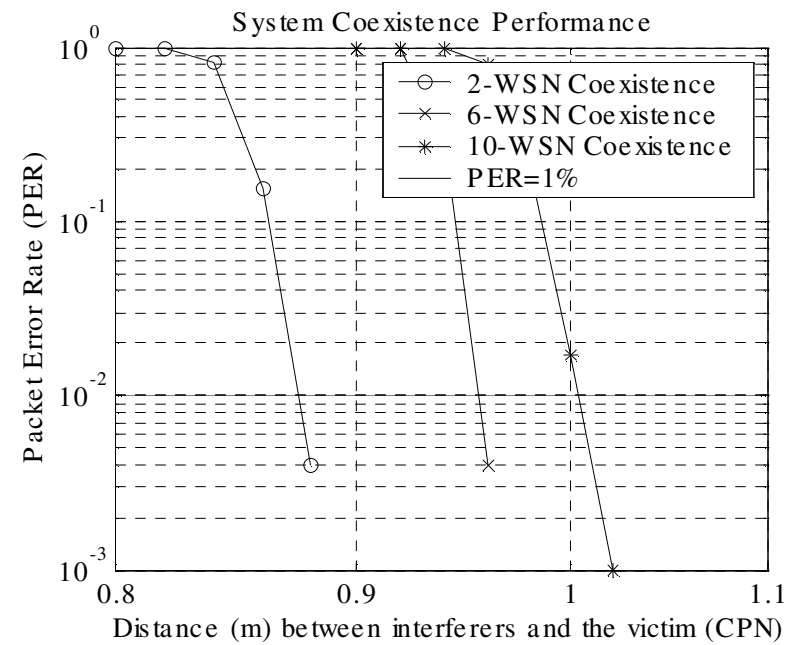

Fig. 5. The resulting packet error rate performance under different number $(\mathrm{N})$ of WSN coexistence in variant interference distance. Among the WSNs, the (N-1)-WSNs act as interferers to a remote CPN.

operated in the WMTS band, the only interferers come from themselves (WSNs). Since the CDMA is provided for signal identification in an interference-free band, the system is able to maintain a high performance in a short-distance interferer. This satisfies the application requirements in the WBAN system.

In the healthcare applications, system performance is restricted in a lower error rate, and a PER less than $1 \%$ is required. In the quantization error analysis, Fig. 6 shows the performance comparison in different DAC word-length in a transmitter. Here we allow an implementation loss less than $0.3 \mathrm{~dB} \mathrm{SNR}$, and the corresponding fewest necessary word-length is 6 bits. According to the sampling rate and dynamic range, a budget of system power consumption could be defined. From the state-of-the-art data conversion technologies [9][10] and RF circuit design [11], maximum 
allowable power consumption is defined and limited within $200 \mathrm{uW}$ and $300 \mathrm{uW}$ (after dynamic range and bandwidth scaling), respectively. In the baseband, a fully circuit is designed with HDL code. After circuit synthesis and place-route, the simulated power is $50 \mathrm{uW}$. As a result, the overall system power is bounded in $550 \mathrm{uW}$.

In Table I, the key features of some possible systems for healthcare applications are listed and compared. In signal modulation, the MT-CDMA presents a higher reliability in body signal monitoring. The operating center frequency is located in the WMTS band, which has least interference from consumer electronics and home devices. In the allowable $3 \mathrm{MHz}$ bandwidth, we could reach a maximum data rate of 86 $\mathrm{kb} / \mathrm{s}$ when the time-spreading, frequency-spreading, and the overhead of guard interval are considered together. With the specified 550uW power budget, we have least energy-bandwidth product per bit, say 0.0192 . In other words, our system requires the least processing energy and signal bandwidth to deliver one bit. Finally, we summarize the proposed system specification in Table II.

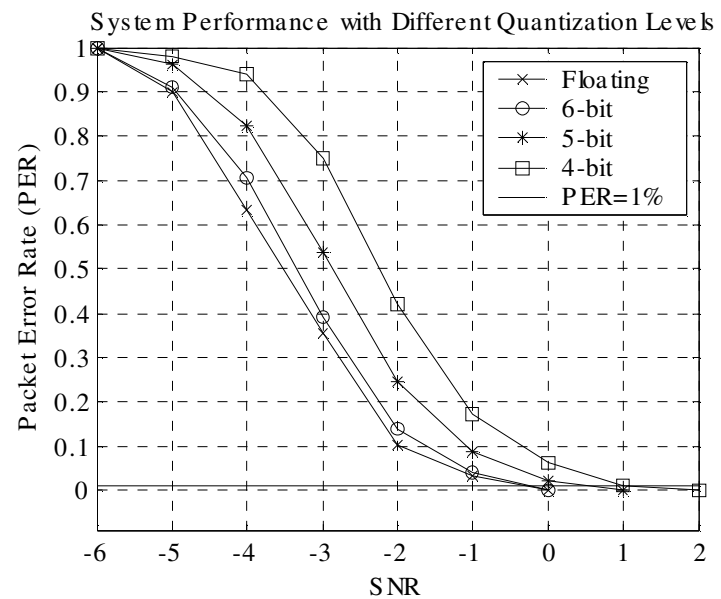

Fig. 6. The packet error rate performance of single proposed MT-CDMA sensor unit with different data quantization levels.

TABLE I

\begin{tabular}{ccccc}
\hline \hline & Proposed & $\begin{array}{c}{[1]} \\
\text { ZigBee }\end{array}$ & $\begin{array}{c}{[2]} \\
\text { IR-UWB }\end{array}$ & $\begin{array}{c}{[3]} \\
\text { BT }\end{array}$ \\
\hline \multirow{2}{*}{ Modulation } & MT- & BPSK & PPM & FHSS \\
& TDM & O-QPSK & & \\
Center Freq.(fc) & $1.4 \mathrm{G}$ & $868 \mathrm{M} / 915 \mathrm{M} / 2.4 \mathrm{G}$ & $3 \sim 4 \mathrm{G}$ & $2.4 \mathrm{G}$ \\
Radio BW & (WMTS) & (ISM) & $($ ISM) & $($ ISM) \\
Data Rate & $86 \mathrm{k}$ & $300 \mathrm{k} \sim 2 \mathrm{M}$ & $528 \mathrm{M}$ & $1 \mathrm{M}$ \\
Sensor Nodes & $\sim 10$ & $250 \mathrm{k}$ & $100 \mathrm{k}$ & $250 \mathrm{k}$ \\
Total Power & $550 \mathrm{u}$ & $\sim 254$ & $\sim 10$ & $\sim 7$ \\
Energy*BW & 0.0192 & $50 \mathrm{~m}$ & $1 \mathrm{~m}$ & $150 \mathrm{~m}$ \\
(mJ*MHz/bit) & & 0.06 & 5.28 & 0.6
\end{tabular}

Key features and comparisons with the candidate systems for healthcare applications.

BT: Bluetooth
TABLE II

\begin{tabular}{cc}
\hline \hline RF Band & $1395 \sim 1400 \mathrm{MHz}$ \\
DAC Wordlength & 6 \\
Maximum Data Throughput & $86 \mathrm{~kb} / \mathrm{s}$ \\
User Spreading Code Type & Gold Code \\
Spreading Code Length & 31 \\
Spreading Code Chip Rate & $3 \mathrm{Mc} / \mathrm{s}$ \\
IFFT/FFT Block Size & 16 \\
Guard Interval Duration & $20.6 \mathrm{us}$ \\
Constellation Mapper & QPSK \\
\hline \hline
\end{tabular}

The specification summary of the proposed MT-CDMA based WBAN system

\section{CONCLUSION}

This work presents an MT-CDMA based WBAN system with the most efficient energy-spectrum product compared to the state-of-the-art systems for healthcare applications. The power budget, coexistence capability, and PAPR problems are considered and reflected in the system specification. With the proposed work, we provide an alternative solution with higher reliability and flexibility for ubiquitous healthcare and WBAN applications.

\section{REFERENCES}

[1] Wireless Medium Access Control (MAC) and Physical Layer (PHY) Specifications for Low-Rate Wireless Personal Area Networks (LR-WPANS), IEEE Standard 802.15.4, 2003

[2] J. Rychaert, et. Al, "Ultra-Wideband Transmitter for Low-Power Wireless Body Area Networks: Design and Evaluation," IEEE Trans. Circuits and System I, vol. 52, no. 12, pp. 2515-2525, Dec. 2005.

[3] Wireless Medium Access Control (MAC) and Physical Layer (PHY) Specifications for Wireless Personal Area Networks (WPANS), IEEE Standard 802.15.1, 2005.

[4] Wireless Medium Access Control (MAC) and Physical Layer (PHY) Specifications: High-speed Physical Layer in the 5GHz Band, IEEE Standard 802.11a, 1999.

[5] Federal Communications Commission, "Amendment of Parts 2 and 95 of the Commission's Rules to Create a Wireless Medical Telemetry Service," FCC Washington, D.C., Rep. FCC00-211, 2000.

[6] R. Gold, "Optimal binary sequences for spread spectrum multiplexing," IEEE Trans. Information Theory, vol. 13, no. 4, pp. 619-621, Oct. 1967.

[7] Y. Kou, Wu-Sheng Lu, and A. Antoniou, "New peak-to-average power-ratio reduction algorithms for multicarrier communications," IEEE Trans. Circuits and Systems I, vol. 51, no. 9, pp. 1790-1800, Sept. 2004.

[8] A. Aggarwal and T.H. Meng, "A convex interior-point method for optimal OFDM PAR reduction," in Proc. 2005 IEEE Int. Conf. Communications, pp. 1985-1990.

[9] K.L. Chan and I. Galton, "A 14b 100MS/s DAC with Fully Segmented Dynamic Element Matching," in Proc. 2006 IEEE Int. Solid-State Circuit Conf. (ISSCC), pp. 582-583, Feb. 2006.

[10] J.A.M. Jarvinen, Mikko Saukoski, and K. Halonen, "A 12-bit 32uW Ratio-Independent Algorithmic ADC," in Proc. 2006 IEEE Symposium VLSI Circuits, pp. 58-59, Jun. 2006.

[11] Y.H. Chee, A.M. Niknejad, J. Rabaey, "A $46 \%$ Efficient $0.8 \mathrm{dBm}$ Transmitter for Wireless Sensor Networks," in Proc. 2006 IEEE Symposium VLSI Circuits, pp. 52-53, Jun. 2006.

[12] J.Y. Yu, et. Al, "A $31.2 \mathrm{~mW}$ UWB Baseband Transceiver with All-Digital I/Q mismatch Calibration and Dynamic Sampling," in Proc. 2006 IEEE Symposium VLSI Circuits, pp. 290-291, Jun. 2006.

[13] R. Prasad and S. Hara, "Overview of multicarrier CDMA," IEEE Communications Magazine, pp. 126-133, Dec. 1997. 